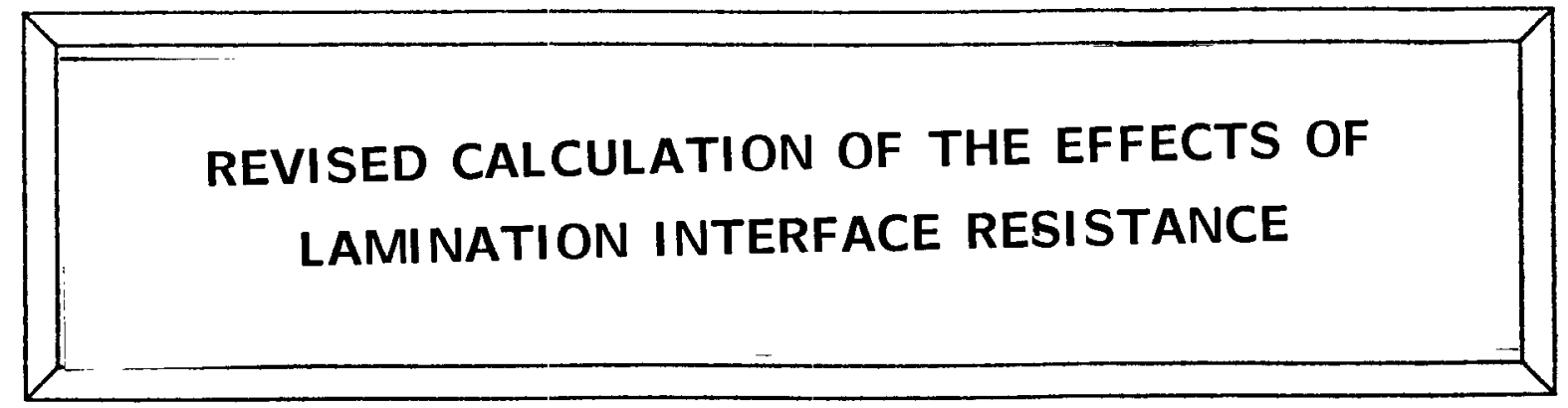

AD

Booster Technical Note

No. 68

G, H, MORGAN

NOVEMBER 10, 1986

ACCELERATOR DEVELOPMENT DEPARTMENT

Brookhaven National Laboratory

Upton, N.Y. 11973 


\section{Revised Calculation of the Effects of Lamination Interface Resistance}

As initially reported ${ }^{(1)}$, the computation of currents crossing between laminations assumed a uniform conductance distributed across the lamination interface. The edge current was assumed to occupy the same fraction of the width as of the thickness. This analysis was recently made use of to estimate the effect of stamping burrs (2). An analysis of two-dimensional eddy currents in a single lamination was also just completed ${ }^{(3)}$ and it shows that the edge curent in a poleface lamination occupies only about a skin depth (typically $1.5 \mathrm{~mm}$ ). The time constant for flux penetration at the edges is typically 25 second.

It is now assumed that a uniform interface conductance will permit current crossing from one lamination to the next only in a small region at the edges also. In effect, this raises the ac interface resistance because the current path has a smaller cross-section. The lamination thickness is assumed to be less than a skin depth, and a steady state current profile through the lamination thickness is assumed, i.e. $J=z \dot{B}$. Both the edge current and the interlamination current are assumed to occur in a thickness of one skin depth. Figures 1 and 2 from reference (2) are reused here. The resistances of Fig. 2, for a lamina of height $h$ meter, width $w$ and thickness $\mathrm{t}$ are $\mathrm{r}_{1}=2 \rho \mathrm{w} / \mathrm{th}, \mathrm{r}_{2}=\rho \mathrm{t} / 2 \mathrm{~h} \delta, \mathrm{r}_{3}=\rho_{\mathrm{S}} / \mathrm{h} \delta$ or $\mathrm{r}_{3}=\rho_{\mathrm{e}} / \mathrm{h}$, where $\rho$ is the resistivity of the lamination, $\delta$ the skin depth, $\rho_{S}$ the surface resistivity and $\rho_{e}$ the edge resistivity. These 2 latter quantities are explained below.

If there are $n$ laminations, there are $m=2 n-1$ current loops (Fig. 2 is the 2-lamination diagram), alternately a lamination loop and an interface loop. As in ref (1), because of symmetry, $I_{k}=I_{m-k+1}$, where the subscript denotes loop current, so there are only $n$ unknown currents and $n$ equations. A typical lamination equation is

$$
-r_{1} I_{j-1}+2\left(r_{11}+r_{2}\right) I_{j}-r_{1} I_{j+1}=\epsilon
$$

and a typical interface equation is

$$
-r_{1} I_{j-1}+2\left(r_{1 !}+r_{3}\right) I_{j}-r_{1} I_{j+1}=\epsilon
$$

where $E=w t \dot{B} / 2$. Note that the interface equation does not have zero emf, contrary to the analysis presented in reference (1), where the various currents were assumed to exist in an infinitesimally thick sheet. The first equation $(\mathrm{j}=1)$ is a lamination equation and is

$$
2\left(\mathrm{r}_{1}+\mathrm{r}_{2}\right) \mathrm{I}_{1}-\mathrm{r}_{1} \mathrm{I}_{2}=E
$$

If $\mathbf{n}$ is odd the final equation, $\mathrm{j}=\mathrm{n}$, is a lamination equation and is

$$
-2 r_{1} I_{n-1}+2\left(r_{1}+r_{2}\right) I_{n}=\epsilon
$$

If $\mathrm{n}$ is even, the final equation is an interface equation and is

$$
-2 r_{1} I_{n-1}+2\left(r_{1}+r_{3}\right) I_{n}=E
$$

The set of $\mathrm{n}$ tridiagonal equations is solved using the Thomas algorithm. 
The data used for the following computations are based on silicon steel of thickness $t=26$ mil, resistivity $\rho=30 \mu \Omega . c m$, and relative permeability 4800. The pole face width $w$ is $10 \mathrm{inch}, \dot{B}=6.94 \mathrm{~T} / \mathrm{sec}$ and the frequency is 7.5 Hz. In reference (3), Cottingham gives interface resistances in an asreceived stack of 28 laminations arranged in a repeated back-back, backfront, back-front, front-front sequence. (The back has the burr). These laminations have an area of $0.312 \mathrm{~m}^{2}$ and a total periphery of $4.12 \mathrm{~m}$. The mean front-front resistance is $125 \pm 62 \mathrm{~m} \Omega$, the mean front-back resistance is $20 \pm 14 \mathrm{~m} \Omega$ and the mean back-back resistance is $0.31 \pm .09 \mathrm{~m} \Omega$. If it is assumed that the surface resistance, given by the front-front average, is in parallel with the edge resistance, and that the parallel combination is given by the front-back average, then the edge resistance is $24.2 \mathrm{~m} \Omega$. The average surface resistivity $\rho_{S}$ is resistance times area or $0.039 \Omega . \mathrm{m}^{2}$ and the average edge resistivity $\rho_{\mathrm{e}}$ is resistance times edge length or $0.100 \Omega . \mathrm{m}$ in the front-back case and $1.3 \mathrm{~m} \Omega . \mathrm{m}$ in the back-back case.

The numerical calculations were made for both cases: one assuming only the surface resistance is present, the other assuming only the edge (burr) resistance is present. For the former case, in order to get an interlamination current equal to $1 / 10$ the lamination current, the surface resistivity must be $\rho_{\mathrm{S}}=12.1 \mu \Omega . \mathrm{m}^{2}$. The previous finding $(1)$ was $450 \mu \Omega \mathrm{m}^{2}$. In the second case, the edge resistivity required for the same 10:1 current ratio is $\rho_{\mathrm{e}}=4.16 \mathrm{~m} \Omega . \mathrm{m}$. Both numbers are less than the average measured values, and even the average burr-to-burr contact, with $\rho_{\mathrm{e}}=1.3 \mathrm{~m} \Omega . \mathrm{m}$ is only a factor of 3 too low - it gives an interface current which is $26 \%$ of the lamination current.

The conclusion is that using the laminations in the as-received condition should be satisfactory, if one allows rms averaging of the effects as argued in ref (2).

\section{References}

(1) G. Morgan "Effect of Interface Resistance Between Magnet Laminations", Booster Tech Note 29, 4/30/86

(2) G. Cottingham, G.H. Morgan, W.L. Stokes, "The Effect of Stamping Burrs on Interlamination Resistance", Booster Tech Note 65, 10/24/86

(3) G.H. Morgan, "Magnet lamination Eddy Currents Reexamined", Booster Tech Note $67,11 / 4 / 86$ 


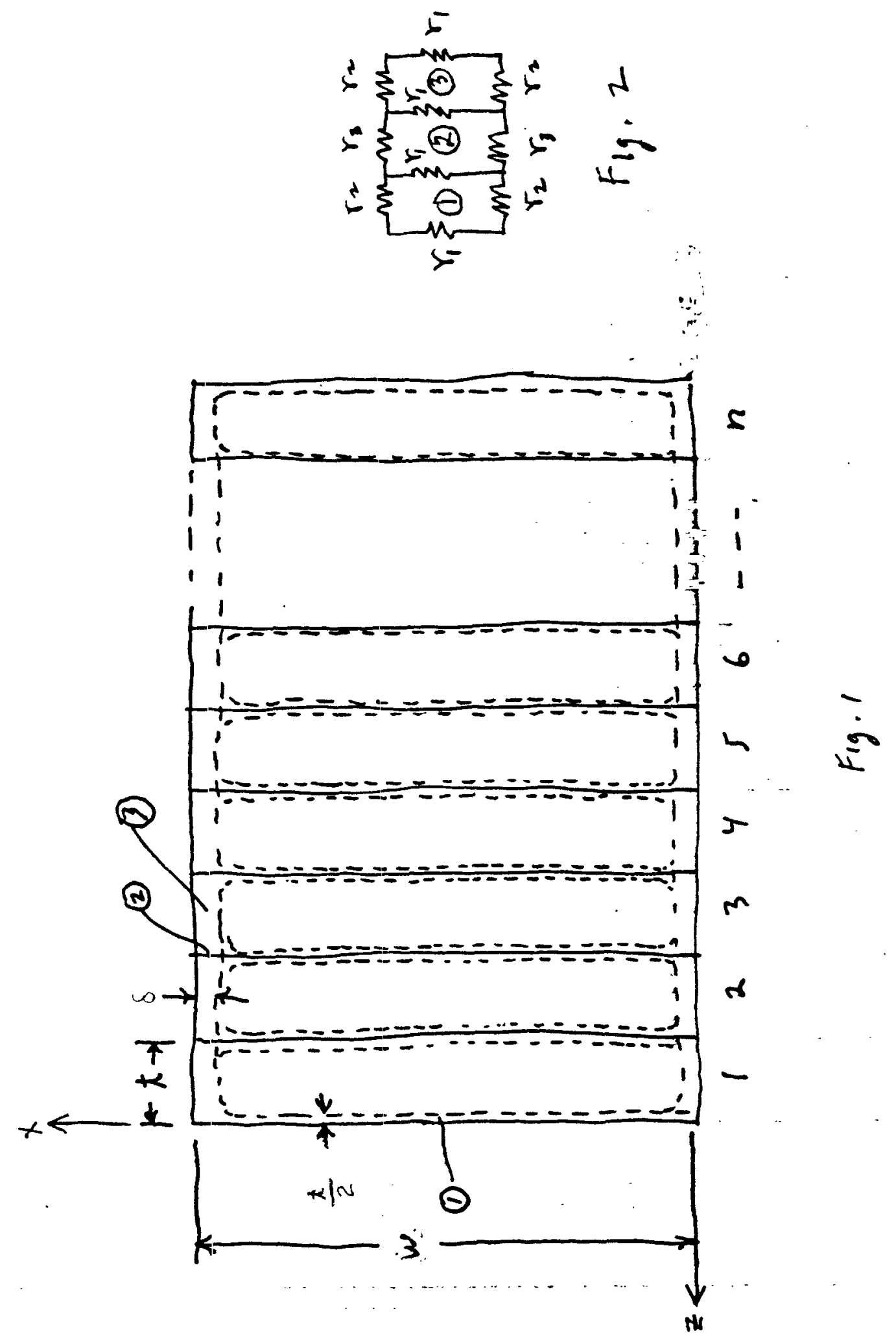

